\title{
Acolhimento e cuidado à pessoa em uso problemático de drogas
}

\author{
Embracing and caring for problematic drug users \\ Acogimiento y cuidado a la persona en uso problemático de drogas
}

\author{
Maria de Fátima Alves Aguiar Carvalho'; Edméia de Almeida Cardoso Coelho"; Jeane Freitas de Oliveira"I, \\ Ana Karla da Silva Freire ${ }^{\prime V}$; Andiara Rodrigues Barrosv, Rosália Teixeira Luz ${ }^{\text {VI }}$
}

\begin{abstract}
RESUMO
Objetivo: analisar práticas de acolhimento na atenção à pessoa em uso problemático de drogas sob o enfoque da integralidade. Método: estudo qualitativo, tendo integralidade como categoria analítica, realizado com 14 usuários e nove profissionais de um Centro de Atenção Psicossocial em 2015, Pernambuco/Brasil. A pesquisa foi aprovada pelo Comitê de Ética em Pesquisa. $O$ material empírico foi produzido por meio de quatro oficinas de reflexão e analisado pela técnica de análise de discurso. Resultados: os discursos de usuários do serviço e de profissionais convergem para práticas de cuidado acolhedoras, capazes de construir relações de confiança e vínculos consistentes, facilitando processos terapêuticos caracterizados pelos dois grupos como resolutivos. Conclusão: $\mathrm{O}$ cuidado prestado a pessoas em uso problemático de drogas caminha ao encontro da integralidade, com práticas de acolhimento qualificadas como dialógicas, afetivas e potencial para resolutividade em um contexto favorável à autonomia e ao fortalecimento da cidadania.
\end{abstract}

Descritores: Integralidade em saúde; serviços de saúde mental; usuários de drogas; transtornos relacionados ao uso de substâncias.

\section{ABSTRACT}

Objective: with a comprehensive care focus, to examine practices in care for problematic drug users. Method: this qualitative study of 14 users and nine health professionals at a Psychosocial Care Center in Pernambuco, Brazil, in 2015, was approved by the research ethics committee. The empirical material was produced at four reflection workshops, and analyzed by discourse analysis. Results: the service users' and health personnel's discourses converged towards welcoming care practices able to build relationships of trust and lasting bonds, thus facilitating therapeutic processes that both groups characterized as producing solutions. Conclusion: the care provided to problematical drug users is progressing towards comprehensiveness, by way of user embracement practices that users and professionals describe as dialog-based, affective and potentially leading to solutions in a context favorable to autonomy and stronger citizenship.

Descriptors: Integrality in health; mental health services; drug users; substance-related disorders.

\section{RESUMEN}

Objetivo: analizar prácticas de acogida en la atención a la persona en uso problemático de drogas bajo el enfoque de la integralidad. Metodología: estudio cualitativo, siendo que la categoría analítica es la integralidad, realizado junto a 14 usuarios y nueve profesionales de un Centro de Atención Psicosocial en 2015, Pernambuco/Brasil. La investigación fue aprobada por el Comité de Ética de Investigación. El material empírico fue producido por medio de cuatro talleres de reflexión y analizado mediante la técnica de análisis de discurso. Resultados: los discursos de los usuarios del servicio y de profesionales convergen hacia prácticas de cuidado acogedoras, capaces de construir relaciones de confianza y vínculos consistentes, lo que facilita procesos terapéuticos caracterizados por los dos grupos como siendo resolutivos. Conclusión: El cuidado ofrecido a personas con uso problemático de drogas va hacia la integridad, con prácticas de acogida calificadas como siendo dialógicas, afectivas y con potencial para resolución en un contexto favorable a la autonomía y al fortalecimiento de la ciudadanía.

Descriptores: Integralidad em salud; servicios de salud mental; consumidores de drogas; trastornos relacionados con sustancias.

\section{INTRODUÇÃO}

A implementação da Reforma Psiquiátrica vem, desde o final da década de 70, alterando acepções e práticas em saúde mental que incidem também nas pessoas que fazem uso de drogas. Essa mudança tem suscitado novos paradigmas de atenção e, consequentemente, novos modos de cuidar. Embora com muitos entraves, o Brasil tem sido referência pela Organização das Nações Unidas (ONU) inclusive, para países de primeiro mundo ${ }^{1}$.

'Enfermeira. Doutora. Professora Adjunta. Universidade Federal do Vale do São Francisco. Petrolina, PE, Brasil. E-mail: fatimaaguiar@hotmail.com.br "Enfermeira. Doutora. Professora Associada. Universidade Federal da Bahia. Salvador, BA, Brasil. E-mail: edmeiacoelho@gmail.com

I'Enfermeira. Doutora. Professora Associada. Universidade Federal da Bahia. Salvador, BA, Brasil. E-mail: jeane.foliveira@outlook.com

IVEnfermeira. Mestranda. Universidade de Pernambuco. Recife, PE, Brasil. E-mail: akarlasf@hotmail.com

VEnfermeira. Doutoranda. Universidade Federal da Bahia. Salvador, BA, Brasil. E-mail: andiarabarros@gmail.com

v'Enfermeira. Doutora. Professora Adjunta. Universidade Estadual do Sudoeste da Bahia. Jequié, BA, Brasil. E-mail: rosluz@gmail.com

vilsíntese da tese de doutorado Integralidade do Cuidado na Atenção Psicossocial, 2016, Programa de Pós-Graduação em Enfermagem, Universidade Federal da Bahia, Brasil. 
Entretanto, novo cenário se anuncia sem o necessário debate com a sociedade, representando retrocessos e comprometendo a lógica organizativa do cuidado compartilhado em rede. O Ministério da Saúde (MS) vem editando regulamentos que contrariam recomendações da Lei $\mathrm{n}$ - $10.216 / 2001$, que tem no acolhimento sua mais potente ferramenta de cuidado.

O acolhimento se destaca na reorganização da Política de Saúde Mental, visto que tem embasado mudanças nas práticas relacionais com foco na pessoa e na superação do modelo centrado na doença e nas relações verticalizadas que teimam em subsistir nos serviços de saúde ${ }^{2}$. Assim, requer integração de ações de promoção, prevenção, tratamento e reabilitação, promovendo atenção nos diferentes níveis de percurso da rede assistencial na perspectiva da integralidade.

$\mathrm{Na}$ atenção às pessoas que usam drogas, a integralidade compreende uma pluralidade de ações que compõem projetos terapêuticos singulares, elaborado por equipes colaborativas. Essa aposta contempla regulação das políticas públicas, reorientação das relações entre o Estado e a sociedade e enxergar o usuário sob uma lógica de atendimento despida de preconceitos, que considera o cuidado em sua multiplicidade ${ }^{2}$.

Nessa concepção, o Centro de Atenção Psicossocial Álcool e outras Drogas (CAPSad) solicita planos terapêuticos compartilhados entre profissionais, usuários e familiares, com base na valorização de processo de trabalho menos burocrático, que respeita à diversidade e à singularidade, no encontro que possibilita compreender a pessoa e suas demandas, considera experiências e atenta para suas necessidades ${ }^{3}$.

Nesse (re)desenho do cuidado, o acolhimento é o intercessor, que sustenta relação de confiança entre todos os envolvidos, propiciando uma atenção colaborativa e responsabilizada ${ }^{4}$. Em suma, adquire espaço importante no cenário nacional das políticas públicas, com promoção de práticas que possibilitam o acesso e a acessibilidade, incentivam ao autocuidado e promovem maior visibilidade a enfermagem e demais profissionais não médicos ${ }^{4}$.

Em pesquisa que estudou o cuidado em CAPSad, a integralidade constituiu perspectiva que orienta práticas interprofissionais e o acolhimento constitui-se dispositivo que imprime transformações às práticas, produz e consolida vínculos entre profissionais, usuários e familiares no encontro de quem cuida e de quem é cuidado. Divulgamos neste artigo categoria empírica da referida pesquisa, em que o acolhimento se expressa como dimensão da integralidade, tendo como objetivo analisar práticas de acolhimento na atenção à pessoa em uso problemático de drogas sob o enfoque da integralidade.

\section{REVISÃO DE LITERATURA}

Acolhimento implica em postura ética e cumplicidade interprofissional em consonância com as necessidades das pessoas, dos coletivos e das populações. Destarte, não estaciona em um único serviço ou trabalhador, deve estar presente em todos os percursos da rede de atenção ${ }^{5}$. Por isso, é considerado dispositivo dinamizador e transformador, que intermedia ações, qualifica profissionais e possibilita ao usuário o seu protagonismo ${ }^{6}$.

O acolhimento consolida-se no quadrilátero que potencializa encontros: escuta, vínculo, responsabilização e resolutividade, dimanando a qualidade da atenção. É o dispositivo medianeiro que visa à produção de relações intersubjetivas de confiança na constituição de compromissos, concretizada no cuidado colaborativo entre profissionais, serviços de saúde e demais dispositivos ${ }^{7}$, efetivando a integralidade.

Integralidade, a princípio, diz respeito à assistência integral e a forma dos serviços de saúde se organizar. Essas noções acabaram ganhando outras dimensões que envolvem saberes e práticas profissionais, estratégias políticas em defesa do Sistema Único de Saúde (SUS), qualidade da atenção, legitimidade social e sustentabilidade ${ }^{8}$, na construção de um cuidado efetivo à saúde.

Nessa circunstância, o Centro de Atenção Psicossocial (CAPS) incorpora ações que objetivam inclusão social, protagonismo, laços sociais e estimula potencialidades. Propõe-se a prestar cuidado a pessoas com transtornos mentais e decorrentes do uso de álcool e outras drogas, com base na atenção psicossocial orientada pela estratégia de Redução de Danos $(R D)^{9}$. Esse serviço tornou-se referência de cuidado em saúde mental por estabelecer a reorganização da rede assistencial, a partir de uma lógica territorial ${ }^{10}$, que requer dispositivos na comunidade para compor e articular as múltiplas nuances de cuidado na perspectiva da integralidade.

$\mathrm{Na}$ atenção psicossocial os dispositivos que compõem a Rede de Atenção Psicossocial (RAPS) são articulados e profissionais são responsáveis pela resolubilidade das demandas, seja na atenção primária, no CAPS, no hospital de referência ou em outros serviços, desconstruindo a lógica de referência e contra referência, que favorece a desresponsabilização e dificulta o acesso da população ${ }^{11}$. 


\section{MEtodologia}

Estudo descritivo de abordagem qualitativa, tendo integralidade como categoria analítica, realizado entre março e agosto de 2015, em um CAPSad de Pernambuco/Brasil.

Colaboraram com a pesquisa 14 usuários e nove profissionais. Para o grupo de usuários os critérios de inclusão foram: ter vínculo com o CAPSad há pelo menos três meses e apresentar condições de discernimento sobre o tema conforme os objetivos propostos na pesquisa.

Da equipe, composta por 14 profissionais, nove atenderam aos critérios de inclusão, que foram: atuar há mais de cinco meses na unidade e não estar afastado por licença médica ou prêmio. 0 tempo de cinco meses foi estabelecido por considerarmos adequado à devida inserção na rotina do serviço, bem como para garantir a participação de todas as categorias.

O material empírico foi produzido por meio de quatro oficinas de reflexão, duas com cada grupo de participantes, denominadas Sentidos do Cuidado no CAPSad e Resgatando o cuidado em CAPSad.

As oficinas permitem emergir emoções por vezes adormecidas ou desconhecidas, desde que transcorram em clima acolhedor e de respeito, articula o que os participantes pensam, sentem e fazem em torno de uma questão central inserida em um contexto social ${ }^{12,13}$.

A primeira oficina com usuários foi desenvolvida a partir das questões reflexivas: Como eu me sentia ao chegar no CAPSad? Que experiência de cuidado vivenciei desde a minha chegada? Como essas experiências têm afetado a minha vida?

Na primeira oficina com profissionais partimos das questões reflexivas: Quem é a pessoa que frequenta o CAPSad? Que experiências tenho vivenciado aqui? Tenho atendido às necessidades dessa pessoa?

Na segunda oficina, em ambos os grupos, resgatamos os aspectos valorizados pelos participantes no primeiro encontro, submetido à apreciação. Houve concordância dos conteúdos expostos e foram acrescentados novos elementos a partir da reflexão e discussão ao responderem à questão: vocês se sentem contemplados na síntese da oficina anterior?

As oficinas foram conduzidas por facilitadora e colaboradora, duraram em média duas horas. Obedeceram às etapas: apresentação, desenvolvimento, socialização, síntese e avaliação ${ }^{12}$. Foram gravadas e posteriormente transcritas.

Para análise do material empírico, foi utilizada a técnica análise de discurso segundo Fiorin. A análise de discurso é uma prática e um campo da linguística e da comunicação especializada em analisar construções ideológicas presentes num texto.Foram seguidas as etapas propostas pelo autor: leitura do texto localizando as figuras e temas que conduziram a bloco de significação; depreensão de temas parciais e organização por convergência em planos de significado, construção de subcategorias e respectivas categorias empíricas centrais ${ }^{14}$.

A pesquisa observou a Resolução no 466/2012 e foi aprovada pelo Comitê de Ética sob parecer no 0008/141014. Para garantir o anonimato, os colaboradores foram identificados segundo a condição de usuário (U1...) ou profissional (P1...).

\section{RESULTADOS E DISCUSSÃO}

Do grupo de usuários, a maioria era do sexo masculino, casada, ensino fundamental e média de idade de 42 anos. O tempo no CAPSad variou de 5 meses a 9 anos. Sobre o uso de drogas, seis afirmaram usar exclusivamente o álcool e os demais referiram usar duas ou mais substâncias, prioritariamente maconha, cocaína e crack.

Do grupo de profissionais, colaboraram com a pesquisa: assistente social, enfermeira, médico psiquiatra, farmacêutica, psicóloga (2), recepcionista, porteiro e cozinheira. A maioria era do sexo feminino, solteira, com média de idade de 38 anos. 0 tempo no serviço variou de 5 meses a 9 anos.

As duas categorias temáticas emergentes da análise dos discursos dos colaboradores são tratadas a seguir.

\section{Escuta sensível potencializando subjetividades}

No CAPSad, pessoas com problemas por uso de drogas passam do sentimento de abandono para o de amparo. Essa condição se materializa no acesso e acessibilidade e no acolhimento por profissionais disponíveis para escuta, representando ruptura com o modelo em que se ergueu a psiquiatria tradicional, cujo foco de interesse era exclusivamente a doença mental. 
A relação profissional usuário, livre de amarras históricas equivocadas, possibilita processos terapêuticos que dão lugar à liberdade, com possibilidades para além de agendas profissionais e muros institucionais, que estimula a autoestima e a autonomia.

Assim, o compromisso e a responsabilidade da equipe em predispor novas perspectivas a pessoas com problemas com drogas, contabiliza desfechos positivos.

A equipe daqui me ajudou, não dava certo em nenhum lugar, já frequentei cinco centros de recuperação. Aqui é um acolhimento de coração, você é respeitado, apesar dos vícios (U1).

Aqui cada funcionário nos faz acreditar em nós mesmos. Percebemos que eles se dão de verdade, porque essa área de saúde mental exige humildade (U4).

Nos encontros entre usuários e profissionais, a escuta permeia as ações de cuidado, sem a imposição de programação, agregando com afeto demandas espontâneas.

Quando eu preciso conversar, eu digo 'doutora, quero conversar com a senhora', saio dali totalmente leve. Eu tenho essa liberdade, a hora que preciso elas tão prontas para ouvir (U3).

Tem situações que me sinto deprimido e eu venho desabafar no CAPS, procuro uma profissional, saio daqui com uma resposta (U2).

Nessa realidade, profissionais adequam protocolos e cronogramas aos contextos de vida das pessoas, reconhecendo questões socioeconômicas e familiares, indo ao encontro da lógica da integralidade, que requer práticas reconstrutivas que facilitem a aproximação de usuários a serviços e a profissionais.

O abarcamento de demandas espontâneas à programação diária traduz a disponibilidade da equipe em garantir o acolhimento. Nessa lógica, a satisfação de usuários com processos terapêuticos, inclui intervenções menos rígidas e burocráticas, que amplia os sentidos do cuidado.

Desse modo, há convergência entre os discursos de usuários e de profissionais à medida em que revelam a disponibilidade da equipe.

Esse acolhimento é importante, às vezes, estamos muito atarefadas e eles querem falar com a gente, e nós ouvimos. É o momento deles se expressarem! (P1).

O CAPS é um local onde a pessoa é escutada, não somente o problema de saúde. É diferente de outro local, o CAPS faz a diferença (P2).

A análise dos discursos de colaboradores revela que o cuidado no CAPSad se dá por vias de atos e afetos, capaz de fortalecer subjetividade, criar vínculo e responsabilização, exercitar o protagonismo de usuários em conformidade que qualifica a atenção e efetiva a integralidade.

\section{Vínculo e responsabilização credibilizando o cuidado}

O respeito e a responsabilização são postos como condições para o êxito das atividades, outorga uma ética definidora de novas práticas que ressignifica o trabalho em equipe e aponta para um horizonte de superação paradigmática.

Eu sou a porta de entrada, tudo passa primeiro lá (portaria), eu recebo eles bem, aí eles entram, se não foram bem atendidos, meu serviço vai por água abaixo (P9).

A colaboração entre profissionais possibilita organização no processo de trabalho, que oferta diferente modalidades/intensidade de cuidado, de acordo com o nível de autonomia e padrão de resposta de cada usuário durante o tratamento ${ }^{8,9}$.

Quando a pessoa opta por intensivo, na maioria das vezes, ela não tem mais vínculos lá fora, geralmente já não trabalha, a família abandonou, então, nós buscamos esses vínculos aqui (P3).

A perda de vínculos sociais em consequência do uso de drogas tira a pessoa do idealizado como refúgio e abrigo emocional. Dessa forma, o encontro com o CAPSad apresenta-se como possibilidade de novos vínculos e novas oportunidades de enxergar a vida, numa experiencia de cidadania que não é mais universal e abstrata.

O uso problemático de drogas é considerado multicausal. Romper esse ciclo é algo muito difícil e delicado, pois as pessoas vivenciam sofrimento físico e psíquico intensos, sendo sua vida afetada em todos os aspectos ${ }^{15}$.

Nessa proposição, a estratégia da RD passa a ser uma oportunidade inovadora de (re)desenhar modos de cuidar, que não exclui outras perspectivas, diante da singularidade de pessoas em sucessivos fluxos terapêutico ${ }^{16}$.

Assim, essa incumbência é assumida com responsabilidade por profissionais que cotidianamente refletem e avaliam possibilidades.

Muitas vezes a pessoa chega e avisa, 'eu não tenho condições de estar aqui todos os dias'. E a função do CAPS é essa, possibilitar todas essas acomodações (P3). 
Eu sempre acho que não estou dando a melhor atenção possível, então às vezes fico pensando, 'será que poderia ter feito algo mais?' (P1).

Cuidar é uma reflexão constante, em alguns momentos, há a impressão de que tudo foi feito, e em outros, de que poderia ter feito um pouco mais (P4).

$\mathrm{O}$ acolhimento constitui-se como vínculo, que influencia positivamente em tratamentos, experienciado quando na presença do profissional, usuários sentem-se resguardados. Essa salvaguarda é expressa por eles, quando em momentos difíceis, a exemplo, na crise de abstinência, tem a certeza que hão de encontrar amparo.

Antes, quando eu entrava em depressão pela falta da droga, procurava me isolar, e agora não, passei o telefone do CAPS para minha esposa, assim fico mais tranquilo (U1).

Nesse diapasão, fortes vínculos e relações de confiança sustentam processos terapêuticos caracterizados como resolutivos, que ficam desestabilizados com o afastamento de profissionais.

A única experiência negativa aqui é quando os profissionais saem, aí deixam uma lacuna muito grande. Porque nós já estamos habituados, já temos uma intimidade. E para reaver essa confiança com outro, vai demorar (U4).

Uma vez construída a relação de confiança estabelece-se também o apego que efetiva o vínculo. Portanto, a saída de profissionais representa ruptura e perda, que repercute em processos terapêuticos vivenciados com sofrimento, por vezes, levando o usuário a abandonar o tratamento.

O apego é entendido como vínculo estabelecido entre uma pessoa e seu principal cuidador. É formado no início da vida, mas se reverbera nos relacionamentos na idade adulta, por meio das distintas funções e relações sociais. 0 conforto experimentado na presença da pessoa de confiança propicia base segura em situações de ameaças, que oferta sentimento de segurança e, alicerça a aliança terapêutica ${ }^{17,18}$.

A ruptura dessa convivência por desligamento do profissional tensiona o relacionamento colaborativo entre equipe e usuário.

A confiança é muito importante, é tanto que quando um profissional sai, eles sentem demais. Aconteceu com a enfermeira antes de mim, quando ela saiu, algumas pessoas se afastaram do CAPS (P1).

A perda do vínculo estabelecido pode modificar a motivação do usuário em relação ao tratamento. Dessa forma, entende-se que os vínculos são importantes para a continuidade do tratamento, uma vez que com ele há a possibilidade de viabilização de espaços de trocas, tão importantes no cuidado em saúde mental ${ }^{19}$. A motivação é referida como um dos mais importantes determinantes no processo terapêutico, cabendo ao profissional proporcionar as condições necessárias para despertar esses afetos ${ }^{20}$, acolhendo empaticamente.

Nosso estudo corrobora pesquisa realizada em Saúde Mental na Espanha, que revela existir no sistema de saúde pública alta probabilidade de que o profissional passe pelo serviço sem concluir o tratamento de algumas pessoas. Essa rotatividade, na maioria das vezes, imprevisível, deixa usuários e profissionais sem a possibilidade de trabalhar o compartilhamento com o outro profissional, acarretando severos prejuízos ${ }^{8}$.

Em situações em que é necessário referenciar usuário a outro serviço, o acolhimento possibilita que o encaminhamento seja compartilhado entre profissionais, usuário, familiares e o serviço que irá recebê-lo, evitando a referência e contrareferência, configurada como repasse de problema 5 .

Na medida em que o CAPSad torna-se o pilar que produz vínculos e superações, poderá também redarguir enclausuramento, motivado pela acomodação do usuário, levando-o ao desinteresse pela alta, em casos que podem ser acompanhados em outros dispositivos da rede.

Aqui é o meu refúgio, onde me achei para me libertar das drogas, gosto de todo mundo, gosto do tratamento, tem uns dez anos que tô aqui (U10).

Tenho oito anos aqui, me sinto bem, todos cuidam de mim, sou bem tratado, os profissionais me ajudam bastante (U11).

Para o alcance da integralidade, que influencia na continuidade do tratamento ${ }^{21}$, outros serviços da RAPS precisam ser acionados, oferecendo suporte ao CAPSad e vice-versa. Os CAPS possuem porta de entrada, entretanto, é determinante que tenham porta de saída, sob o risco de remanicomialização em novos moldes ${ }^{22}$.

\section{CONCLUSÃO}

As práticas de acolhimento à pessoa em uso de drogas foram expressas em duas categorias temáticas emergentes da análise dos discursos dos participantes intituladas: Escuta sensível potencializando subjetividades e Vínculo e responsabilização credibilizando o cuidado. 
O acolhimento é um dispositivo potente às multiplicidades do cuidado no CAPSad, onde a confiabilidade estabelece-se e sustenta-se em processos terapêuticos caracterizados como resolutivos. O compromisso e a disponibilização da equipe em acolher e escutar produz vínculo e horizontaliza relações intersubjetivas.

A satisfação de usuários compreende a acessibilidade a intervenções menos burocráticas, que melhora o processo de trabalho e amplia os sentidos da integralidade. A confiança se constitui na busca de profissionais em reconhecer singularidades e trabalhar as demandas cotidianas segundo as possibilidades do serviço e da equipe, acalmando ansiedades e buscando modos mais efetivos e afetivos de cuidado, que implica flexibilizar protocolos e cronogramas de atividades, fazendo a diferença na atenção psicossocial.

Esta pesquisa amplia o debate e reafirma a atual Política de Saúde Mental, mostrando que é possível produzir resultados positivos em serviços antimanicomiais, inadmitindo, assim, retrocessos pautados em incentivos a tratamentos em Hospitais Psiquiátricos ou Comunidades Terapêuticas que violam direitos, onde pessoas são submetidas a tratamento moral e reclusivos.

$\mathrm{Na}$ incorporação da proposta da integralidade, que precisa ser pensada em rede solidária do cuidado, este estudo apresenta limitação por ter sido desenvolvido em somente um dispositivo da RAPS, o que impede a generalização de seus achados. Entretanto por refletir uma realidade local, serve de estímulo e subsídios para o desenvolvimento de novas pesquisas.

\section{REFERÊNCIAS}

1. Inter-American Drug Abuse Control Commission. Report on drug use in the Americas. OAS Official Records Series; OEA/Ser. L. Washington DC; 2015.

2. Minóia NP, Minozzo F. Admission in Mental Health: Working with Changes in Primary Health Care. Psicol. ciênc. prof. [Internet], 2015 [cited 2019 Mar 10]; 35(4):1340-9. DOI: http://dx.doi.org/10.1590/1982-3703001782013.

3. Jorge MSB, Diniz AM, Lima LL, Penha JC. Matrix support, individual therapeutic project and prodution in mental health care. Texto \& contexto enferm. [Internet], 2015 [cited 2019 Feb 20]; 24(1):112-20. DOI: http://dx.doi.org/10.1590/010407072015002430013.

4. Rocha AS, Spagnuolo RS. User embracement in complex vision: collective action emerging in the Family Health Team. Saúde debate. [Internet], 2015 [cited 2019 Apr 11]; 39(104):124-35. DOI: http://dx.doi.org/10.1590/0103-110420151040394.

5. Dimenstein M, Macedo JP, Gomes M, Silva DM, Abreu MM. Mental Health and Psychosocial Care: Regionalization and Management of Integral on SUS. Salud soc. [Internet], 2018 [cited 2018 Dec 20]; 9(1):70-85. DOI: DOI: http://dx.doi.org/10.22199/S07187475.2018.0001.00004.

6. Constantinidis TC, Cid MFB, Santana LM, Renó SR. Conceptions of Mental Health Professionals about the Therapeutic Activity in the CAPS. Trends Psychol. [Internet], 2018 [cited 2019 Jan 21]; 26(2):911-26. DOI: http://dx.doi.org/10.9788/tp2018.2-14pt.

7. Lopes AS, Vilar RLA, Melo RHV, França RCS. The hospitality in Primary Health Care: relations of reciprocity between workers and users. Saúde debate. [Internet], 2015 [cited 2019 Jul 15]; 39(104):114-23. DOI: http://dx.doi.org/10.1590/0103110420151040563.

8. Kalichman AO, Ayres JRCM. Comprehensiveness and healthcare technologies: a narrative on conceptual contributions to the construction of the comprehensiveness principle in the Brazilian Unified National Health System. Cad. Saúde Pública [Internet] 2016 [cited 2019 May 14]; 32(8):e00183415. DOI: http://dx.doi.org/10.1590/0102-311X00183415.

9. Souza DR, Oliveira MAF, Soares RH, Domanico A, Pinho PH. Resistance of professionals from psychosocial care services in alcohol/drugs whenapproaching harm reduction. J. nurs. health. [Internet], 2017 [cited 2019 Feb 12]; 7(1):16-24. DOI: http://dx.doi.org/10.15210/jonah.v7i1.9276.

10. Pegoraro RF, Nunes LF. Experiences of embracement according to professionals of a psychosocial attention Center. Rev. enferm. atenção saúde [Internet], 2017 [cited 2019 Jan 25]; 6(1):3-17. DOI: http://dx.doi.org/10.18554/reas.v6i1.1525.

11. Nóbrega MPSS, Silva GBF, Sena ACR. Care Network of Psychosocial functioning in Sao Paulo, Brazil: prospects for care in Mental Health. Atas-Investigação Qualitativa em Saúde [Internet], 2016 [cited 2019 Feb 15]; 1(2):41-49. Available from: https://proceedings.ciaiq.org/index.php/ciaiq2016/article/view/735/722.

12. Ferreira ES, Moreira RB, Quadrado JC. Design Women without Frontiers: reflections between theory and practice through the offices "Serial Speech". Rev. cienc. humanas e sociais [Internet], 2016 [cited 2019 Apr 22]; 2(1):70-83. Available from: http://seer.unipampa.edu.br/index.php/missoes/article/view/23048/8689.

13. Spink MJ, Menegon VM, Medrado B. Using workshops as a research strategy: theoretical and methodological articulations and ethical-political applications. Psicol. soc. [Internet], 2014 [cited 2019 May 14]; 26(1):32-42. DOI: http://dx.doi.org/10.1590/S0102-71822014000100005.

14. Fiorin JL. Linguagem e ideologia. São Paulo, Brasil: Editora Ática; 2011.

15. Koob GF, Mason BJ. Existing and future drugs for the treatment of the Dark Side of addiction. Ann. rev. pharmacol. toxicol. [Internet], 2016 [cited 2019 Feb 15]; 1(56):299-322. DOI: https://doi.org/10.1146/annurev-pharmtox-010715-103143.

16. Rameh-de-Albuquerque RC, Lira WV, Costa AM, Nappo SA. From neglection to the actual: the National Drug Users Policy as achievement of the Brazilian Psychiatric Reform. The case of Recife (PE). Psicol. pesq. [Internet], 2017 [cited 2019 May 21]; 1(11):84-96. DOI: http://dx.doi.org/10.24879/2017001100100215. 
17. Scheeren P, Delatorre MZ, Neumann AP, Wagner A. The predictor role of attachment styles in marital conflict resolution. Estud. pesqui. psicol. [Internet], 2015 [cited 2019 Jan 20]; 15(3):835-52. Available from: http://pepsic.bvsalud.org/scielo.php?script=sci_arttext\&pid=S1808-42812015000300004\&lng=pt\&nrm=iso.

18. Bailly J, Taïeb O, Moro MR, Baubet T, Reyre A. "If walls could talk": A photo-elicitation-based observation of service users' perceptions of the care setting and of its influence on the therapeutic alliance in addiction treatment. J. health place [Internet], 2018 [cited 2019 May 24]; 1(54):69-78. DOI: http://dx.doi.org/10.1016/j.healthplace.2018.09.007.

19. Santana TFMC, Pereira MAO. Mental health care in primary care: a cartography. Rev. enferm. UERJ. [Internet], 2018 [cited 2019 May 24]; 26:e32305. DOI: http://dx.doi.org/10.12957/reuerj.2018.32305.

20. Almeida EJ, Leitune CS, Serge ACBP, Terner ML, Silva DAR. Pain and loss: process analysis of mourning. Rev. psicol. IMED. 2015 [cited 2019 May 24]; 7(1):15-22. Available from: https://dialnet.unirioja.es/descarga/articulo/5155064.pdf

21. Queiroz MKS, Rodrigues ILA, Nogueira LMV, Silva IFS. Care flows and comprehensiveness of health care for riverside communities. Rev. enferm. UERJ. [Internet], 2018 [cited 2019 May 24]; 26:e26706. DOI: http://dx.doi.org/10.12957/reuerj.2018.26706.

22. Guimãres TAA, Rosa LCS. The remanicomialization of mental health care in Brazil from 2010 to 2019: analysis of an counterreform conjecture. O Social em Questão [Internet], 2019 [cited 2019 May 20]; 44(1):11-138. Available from: http://osocialemquestao.ser.puc-rio.br/media/OSQ_44_art5.pdf. 